\title{
Niosome Encapsulation of Curcumin: Characterization and Cytotoxic Effect on Ovarian Cancer Cells
}

\author{
Ying-Qi Xu, ${ }^{1}$ Wen-Rong Chen, ${ }^{2}$ Jonathan K. Tsosie, ${ }^{3}$ Xi Xie, ${ }^{4}$ Peng Li, ${ }^{1}$ Jian-Bo Wan, ${ }^{1}$ \\ Cheng-Wei He, ${ }^{1}$ and Mei-Wan Chen ${ }^{1}$ \\ ${ }^{1}$ State Key Laboratory of Quality Research in Chinese Medicine, Institute of Chinese Medical Sciences, University of Macau, \\ Taipa 999078, Macau \\ ${ }^{2}$ College of Traditional Chinese Medicine, Guangdong Pharmaceutical University, Guangzhou 510006, China \\ ${ }^{3}$ David H. Koch Institute for Integrative Cancer Research, Massachusetts Institute of Technology, Cambridge, MA 02139, USA \\ ${ }^{4}$ School of Chemistry and Chemical Engineering, Sun Yat-sen University, Guangzhou 510275, China
}

Correspondence should be addressed to Mei-Wan Chen; mwchen@umac.mo

Received 29 December 2015; Revised 1 May 2016; Accepted 22 May 2016

Academic Editor: Ruxandra Gref

Copyright (C) 2016 Ying-Qi Xu et al. This is an open access article distributed under the Creative Commons Attribution License, which permits unrestricted use, distribution, and reproduction in any medium, provided the original work is properly cited.

Curcumin, a natural chemical compound found in Curcuma longa, has been applied in multiple medicinal areas from antibiotic to antitumor treatment. However, the chemical structure of curcumin results in poor stability, low solubility, and rapid degradation in vivo, hindering its clinical utilization. To address these issues, we have developed a novel niosome system composed of nonionic surfactants: Span 80, Tween 80, and Poloxamer 188. Curcumin was encapsulated in the niosomes with a high entrapment efficiency of $92.3 \pm 0.4 \%$. This system provided controlled release of curcumin, thereby improving its therapeutic capability. Dynamic dialysis was conducted to evaluate the in vitro drug release of curcumin-niosomes. Curcumin-niosomes exhibited enhanced cytotoxic activity and apoptotic rate against ovarian cancer A2780 cells compared with freely dispersed curcumin. These results demonstrate that the curcumin-niosome system is a promising strategy for the delivery of curcumin and ovarian cancer therapy.

\section{Introduction}

Curcumin $\left(\mathrm{C}_{21} \mathrm{H}_{20} \mathrm{O}_{6}\right)$ is a natural yellow compound typically found in Curcuma longa that is regarded as a natural polyphenolic antioxidant presented in many kinds of herbs [1]. Curcumin has been exhibited multiple therapeutic relevance including anticancer, anti-inflammatory, antioxidant, antimicrobial, antirheumatic, and hepatoprotective activities [2]. In particular, the anticancer property of curcumin is responsible for the downregulation of a variety of transcription factors such as AP-1, NF- $\kappa \mathrm{B}$, and $\beta$-catenin $[2,3]$. Its anticancer, anti-inflammatory, antiangiogenic, antineoplastic, and chemosensitizing effects make it a potent candidate in the treatment for multiple types of cancer $[4,5]$. In spite of the well-received pharmacological properties, the therapeutic application of curcumin has been impeded by its shortcomings such as low aqueous solubility at acidic and physiological $\mathrm{pH}$ and its degradability in alkaline conditions $[1,6]$. In addition, poor absorption and rapid metabolism of curcumin severely limit its bioavailability [7]. As a result, researchers have been exploring methods for the effective delivery of curcumin with novel formulations including liposomes [8], micelles [9], conjugates [10], nanoparticles [11], and nanoglobules [12].

Recent developments of nanotechnology and nanomedicine have generated many promising drug delivery systems [13-15]. Niosomes are nonionic surfactant vesicles with a bilayer structure, which have been used to deliver various drug elements including chemotherapeutic agents, genes, hormones, antigens, and peptides [16, 17]. Niosomes share some similarities with liposomes but are composed of nonionic surfactants such as Span 80, Span 60, Span 40, Span 20, Tween 80, and Pluronic 188 instead of phospholipids used in liposomes $[18,19]$. Typically, niosomes are produced from two main components: nonionic surfactants and additives [20]. While nonionic surfactants serve as the vesicular layer, the additives such as cholesterol act to enhance the rigidity 
of the bilayer. This carrier system provides protection of the drug molecule from degradation and inactivation caused by immunological and pharmacological effects. Similar to liposomes, niosomes exhibit superior cellular membrane permeation via osmosis and provide excellent biocompatibility due to their cell membrane-like components. In addition, niosomes surpass liposomes through low production cost and room-temperature chemical storage requirements. In recent years, niosomes are becoming increasingly accepted as an alternative method of drug delivery to liposomes, particularly for drugs with poor stability, low solubility, or rapid degradation [17]. For instance, candesartan cilexetil, a commercially marketed compound that has low bioavailability $(15 \%)$, was loaded inside niosomes composed of Pluronic P85 and Span 60. The niosomes significantly enhanced aqueous solubility and oral bioavailability [21].

The present study seeks to effectively deliver curcumin to tumor sites using niosomes composed of nonionic surfactants Tween 80 and Pluronic 188, in order to improve the solubility and the therapeutic effects of curcumin. The synthesis of curcumin-loaded niosomes (curcumin-niosomes) and the physicochemical properties in terms of particle size, zeta potential, and in vitro drug release were investigated. Furthermore, the in vitro cytotoxicity and therapeutic effects against human ovarian cancer A2780 cell line were evaluated.

\section{Material and Methods}

2.1. Materials. Poloxamer 188 was purchased from BASF SE (Germany). Span 80 and Tween 80 were obtained from Damao Chemical Reagent (Tianjin, China). Poloxamer 188 was obtained from BASF SE (Germany); sodium dodecyl sulfate (SDS) was purchased from Beyotime Biotechnology (Jiangsu, China). Sephadex G50 was obtained from Seebio Biotechnology Co., Ltd. (Shanghai, China). Curcumin was purchased from Huabiao Biotechnology Co., Ltd (Tianjin, China). DMEM culture medium, fetal bovine serum (FBS), penicillin-streptomycin (PS), phosphate-buffered saline (PBS), $0.25 \%(\mathrm{w} / \mathrm{v})$ trypsin/EDTA, propidium iodide (PI), and Hoechst 33342 were obtained from Life Technologies (Grand Island, USA). 3-[4,5-Dimethyl-2-thiazolyl]-2,5diphenyl tetrazolium bromide (MTT) was obtained from Sigma Aldrich (St. Louis, MO, USA). All water used was pretreated with a Milli-Q apparatus (Millipore Corporation, Darmstadt, Germany). All other chemicals used were of analytical grade.

\subsection{Preparation of Curcumin-Niosome. Curcumin-niosomes} were prepared using solvent evaporation. A mixture of surfactants $(0.05 \mathrm{~g}$ of Span $80,0.05 \mathrm{~g}$ of Poloxamer 188, and $0.15 \mathrm{~g}$ of Tween 80 , in a weight ratio of $1: 1: 3$ ) was accurately measured and dissolved in $100 \mu \mathrm{L}$ of anhydrous ethanol. Curcumin $(3.75 \mathrm{mg}$ ) was added to the surfactant solution and mixed thoroughly by ultrasonication and was vortexed at $40-$ $50^{\circ} \mathrm{C}$ such that clear claret-colored miscible substance was obtained. The resultant substance was added drop by drop into $25 \mathrm{~mL}$ preheated $\left(75-80^{\circ} \mathrm{C}\right)$ distilled water. The solution was then mixed via magnetic stirring so that the surfactants were completely dissolved. The hydration process was conducted by stirring the solution for $20-30 \mathrm{~min}$ at $40-50^{\circ} \mathrm{C}$ and the curcumin-niosomes were gradually formed. The niosomes were homogenized under various high-pressure settings to improve niosome nanodispersity while avoiding sample contamination [22].

\subsection{Characterization of Curcumin-Niosome}

2.3.1. Particle Size and Zeta Potential. The average particle size and zeta potential of curcumin-niosome were determined using dynamic light scattering (DLS) Zetasizer using Zetasizer Nano ZSP system (Malvern Instruments, Worcestershire, UK) at $25^{\circ} \mathrm{C}$. Diameter, particle dispersion index (PDI), and zeta potential were obtained based on at least three measurements. The morphology of curcumin-niosome was examined using a transmission electron microscopy (TEM) (JEM-1400, Japan). The sample was deposited on an amorphous carbon-coated copper grid and stained with the phosphotungstic acid solution $(2 \%$, w/v) for $30 \mathrm{~s}$. TEM images were performed at an accelerating voltage of $120 \mathrm{kV}$.

2.3.2. Drug Encapsulation Efficiency (EE\%). The EE\% of curcumin in niosomes was determined using size exclusion chromatography. Microcolumn gel was produced by filling and hydrating Sephadex G50 superfine beads in a $5 \mathrm{~mL}$ syringe (filled to $2.2 \mathrm{~cm}$ ). The microcolumn gel was dehydrated to approximately $0.7 \mathrm{~mL}$ by centrifugation for $2 \mathrm{~min}$ at a rotational speed of $900 \mathrm{rpm}$, forming a $2.0 \mathrm{~cm}$ microcolumn. The sample $(100 \mu \mathrm{L})$ was added to the microcolumn and centrifuged for $2 \mathrm{~min}(900 \mathrm{r} / \mathrm{min})$ to dehydrate the whole $100 \mu \mathrm{L}$ sample. This was followed by repeated eluted processes of $100 \mu \mathrm{L}$ distilled water under the same conditions. The eluate was collected and the niosome concentrations were determined with liquid phase assay. The EE\% of curcumin in curcumin-niosomes was calculated by applying the following equation:

$$
\mathrm{EE} \%=\frac{\left(W_{\text {initial }}-W_{\text {free }}\right)}{W_{\text {initial }}}
$$

2.3.3. In Vitro Drug Release. The release assay was performed to assess the amount of curcumin released from niosomes vesicles. In vitro drug release of curcumin-niosomes was determined using dialysis membrane processing [23]. Curcumin-niosomes $(5 \mathrm{~mL})$ were placed into a preswelled dialysis bag with a molecular weight cut-off at 8,000 Dalton and curcumin was allowed to release into medium composed of $0.5 \%$ of SDS. The medium was incubated at $37^{\circ} \mathrm{C}$ and mixed at $100 \mathrm{rpm}$. Medium samples $(2 \mathrm{~mL})$ were collected after 1, 2, 4, 6, 8, 10, 12, and $24 \mathrm{~h}$ of incubation. Each collected sample was replaced with $2 \mathrm{~mL}$ of fresh medium. The medium samples were promptly filtrated and analyzed using HPLC with a C18 reverse-phase liquid chromatography column $(250 \times 4.6 \mathrm{~mm})$ at a flow rate of $1 \mathrm{~mL} / \mathrm{min}$ at the maximum absorption wavelength of $426 \mathrm{~nm}$ to determine the concentration of released curcumin. The mobile phases consisted of acetonitrile/0.1\% acetic acid (60/40, v/v). Each experiment was repeated in triplicate. 

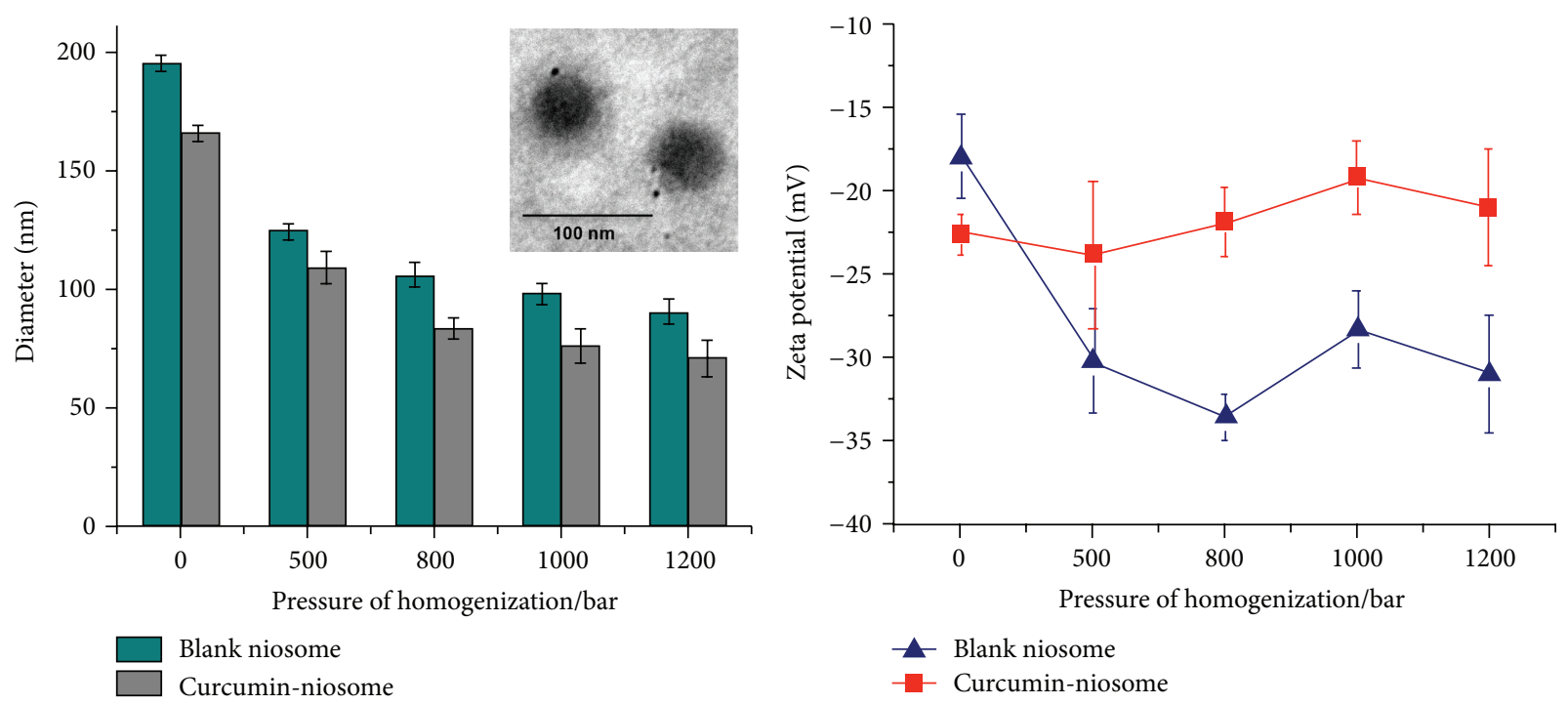

FIGURE 1: Particle size, zeta potential, and TEM image of curcumin-niosome.

2.4. Cell Lines and Cell Culture. Human ovarian cancer A2780 cells were obtained from KeyGen Biotech (Nanjing, China). The cells were cultured in DMEM with $10 \%(\mathrm{v} / \mathrm{v})$ heat-inactivated FBS and antibodies $(100 \mathrm{U} / \mathrm{mL}$ penicillin, $100 \mu \mathrm{g} / \mathrm{mL}$ streptomycin) at $37^{\circ} \mathrm{C}$. The cells were cultured in an incubator with a $5 \% \mathrm{CO}_{2}$ atmosphere at $37^{\circ} \mathrm{C}$ under $5 \%$ $\mathrm{CO}_{2}$.

2.5. Cellular Uptake Study. The native green fluorescence of curcumin and curcumin-niosomes was used to determine cellular internalization in A2780 cells using flow cytometry [24]. A2780 cells were incubated with free curcumin and curcumin-niosomes at a concentration of $12.5 \mu \mathrm{M}$. After incubation for 1,2 , and $4 \mathrm{~h}$, the medium was removed and the cells were carefully washed three times with PBS. The cells were then removed using trypsin and were subsequently washed twice with PBS. The intracellular curcumin fluorescence was quantified with flow cytometry (FACScan, BD, San Jose, CA) [25]. A total of 10,000 cells were collected, amplified, and scaled to generate a single parameter histogram.

2.6. Cell Viability Assay. A2780 cells were seeded in 96-well plates at a density of 5,000 in $100 \mu \mathrm{L}$ of medium per well. When reaching approximately $80 \%$ confluence, the cells were treated with a series of concentrations of free curcumin and curcumin-niosomes for $24 \mathrm{~h}$, respectively. Cell viability was determined with MTT assay, where the cells were incubated with medium containing $1 \mathrm{mg} / \mathrm{mL}$ of MTT for $4 \mathrm{~h}$, followed by dissolving formazan crystals in $100 \mu \mathrm{L}$ of DMSO. Cell viability was evaluated using a $570 \mathrm{~nm}$ microplate reader (SpectraMax M5, Molecular Devices, USA). The results were analyzed according to three independent biological replicates.

2.7. Cell Cycle Analysis. The cell cycle distribution was evaluated as previously described [26]. A2780 cells $\left(2.0 \times 10^{5}\right.$ cells) were treated with free curcumin, blank niosomes without curcumin, and curcumin-niosomes, respectively, and incubated for $24 \mathrm{~h}$. These cells were then harvested, washed twice with PBS for $5 \mathrm{~min}$ by centrifugation, and fixed in $70 \%$ ethanol at $-20^{\circ} \mathrm{C}$ overnight. The collected cells were stained with $100 \mu \mathrm{L}$ of PI stain solution $(30 \mu \mathrm{g} / \mathrm{mL} \mathrm{PI,} 8 \mu \mathrm{g} / \mathrm{mL}$ RNase) for 30 min while protected from light. Analysis was conducted using a flow cytometer (BD FACSCanto ${ }^{\mathrm{TM}}$, BD Biosciences, San Jose, USA). The cell distributions in phases of SubG1, G0/G1, S, and G2/M were calculated using ModFit LT software (version 3.0, Verity, USA). The results were analyzed based on three independent replicates.

2.8. Assessment of Apoptosis. An Annexin V FITC/PI apoptosis detection kit (BD Biosciences) was used to detect cell apoptosis. A2780 cells were exposed to $12.5 \mu \mathrm{M}$ of free curcumin and curcumin-niosomes. After incubation for $24 \mathrm{~h}$, A2780 cells were trypsinized with EDTA-free trypsin and collected by centrifugation, washed twice with cold PBS, and gently resuspended in a binding buffer. The cells were stained with $5 \mu \mathrm{L}$ of Annexin-FITC and $5 \mu \mathrm{L}$ of PI in the dark for $15 \mathrm{~min}$, after which they were analyzed with a flow cytometer (BD Biosciences). All experiments were conducted in triplicate.

2.9. Statistics. The results were expressed as the mean \pm SD. Each value is the mean of at least three independent experiments in each group. Statistical analysis was performed using Student's $t$-test. A significance value of $p<0.05$ was used to indicate significant difference.

\section{Results}

3.1. Characterization of Curcumin-Niosome. The curcuminniosomes were successfully prepared. TEM image showed that curcumin-niosomes had homogeneous spherical shapes and smooth surfaces without aggregation (Figure 1). The diameters of the blank niosomes and the curcumin-niosomes 


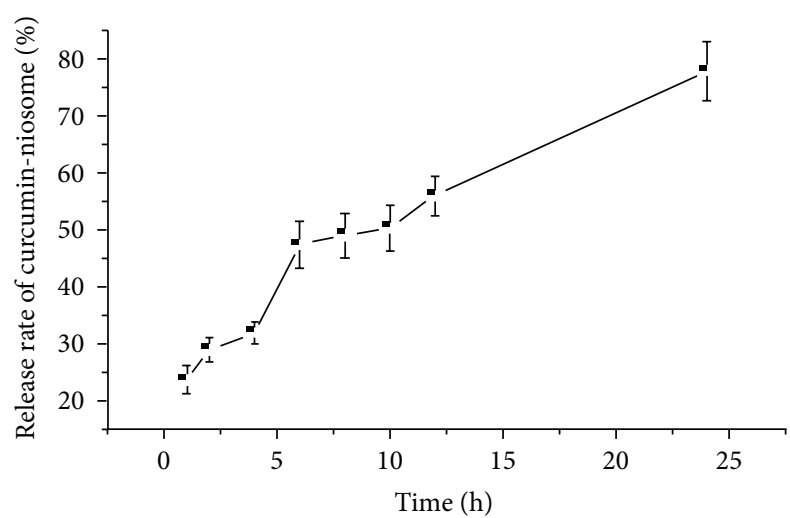

FIGURE 2: Drug release profile of curcumin-niosome in $24 \mathrm{~h}$.

decreased as the pressure of the applied homogenization increased. Interestingly, the diameter and zeta potential of the curcumin-niosomes were smaller than those of the blank niosomes under the same pressure of homogenization (Figure 1). Two reasons may be responsible for this phenomenon. First, curcumin is believed to bind with the lipophilic segments of surfactant, resulting in a more compact curcumin-niosome structure and particle size. This may be due to a favorable lipid-soluble associative region provided by the surfactants. Second, the potential of blank niosomes fluctuated around $30 \mathrm{mV}$, which was related to the high-strength friction during the process of pressure homogenization. However, when curcumin was loaded, the potential value of the particles was slightly decreased, which may be due to the free curcumin distributed in the water phase or potential diffusion layer. The DLS analysis revealed that the mean diameter of curcuminniosomes was $84.15 \pm 4.03 \mathrm{~nm}$ at the homogenization pressure of 800 bar with the drug EE\% of $92.3 \%$, indicating the successful loading of curcumin into niosomes. The size of the niosomes is appropriate for tumor specific accumulation via the enhanced permeability and retention (EPR) effect [27]. Additionally, after niosome storage at $4^{\circ} \mathrm{C}$ for over 3 months, there was less than $20 \mathrm{~nm}$ size increase, suggesting that curcumin-niosomes were stable over storage.

3.2. In Vitro Drug Release Profile. The in vitro release profile of curcumin was investigated via dialysis. The in vitro release behavior of curcumin-niosomes is shown in Figure 2. The release of curcumin from niosomes exhibited a biphasic pattern of constant sustained release after the initial burst. The burst release rate reached $45 \%$ within 6 hours of treatment and decreased thereafter. After $24 \mathrm{~h}$, the accumulated release of curcumin from niosomes reached $80 \%$, suggesting that niosomes provide excellent release from the carriers.

3.3. Cellular Uptake. The uptake of free curcumin and curcumin-niosomes was investigated using flow cytometry. Figure 3 shows the mean fluorescence intensity of A2780 cells treated with either free curcumin or curcumin-niosomes $(12.5 \mu \mathrm{M})$ at different incubation times $(1,2$, and $4 \mathrm{~h})$. After $1 \mathrm{~h}$ of incubation, there is no distinction of cellular uptake between the free curcumin group and curcumin-niosomes group. However, extended incubation periods for 2 and $4 \mathrm{~h}$

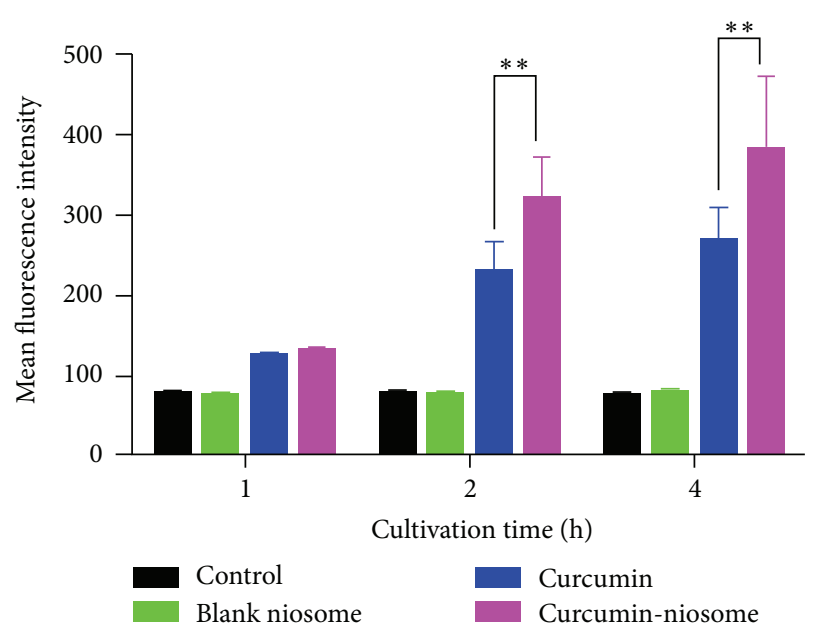

FIGURE 3: Quantification of cellular uptake of curcumin and curcumin-niosomes, ${ }^{* *} p<0.01$.



Figure 4: Cytotoxicity of curcumin-niosomes, ${ }^{*} p<0.05,{ }^{* *} p<$ 0.01 .

showed significant increases of curcumin uptake compared to that of freely dispersed curcumin by A2780 cells. The intracellular levels of curcumin for the curcumin-niosomes group were 1.40-fold and 1.43-fold higher than those of the free curcumin group at $2 \mathrm{~h}$ and $4 \mathrm{~h}$, respectively.

3.4. In Vitro Cytotoxicity of Curcumin-Niosome. The cytotoxicity of curcumin-niosomes in the human ovarian cancer A2780 cell line after $24 \mathrm{~h}$ of incubation was assessed with MTT assay. Compared with free curcumin, curcuminniosomes exhibited significantly higher cell killing rates at all of the tested concentrations (Figure 4). Approximately 50\% of cells survived after treatment with $12.5 \mu \mathrm{M}$ of curcuminniosomes for $24 \mathrm{~h}$, whereas more than $90 \%$ of cells were alive after incubation with free curcumin for $24 \mathrm{~h}$. The cytotoxicity of curcumin-niosomes was more potent (killed > $80 \%$ cells) when higher concentration of curcumin was 

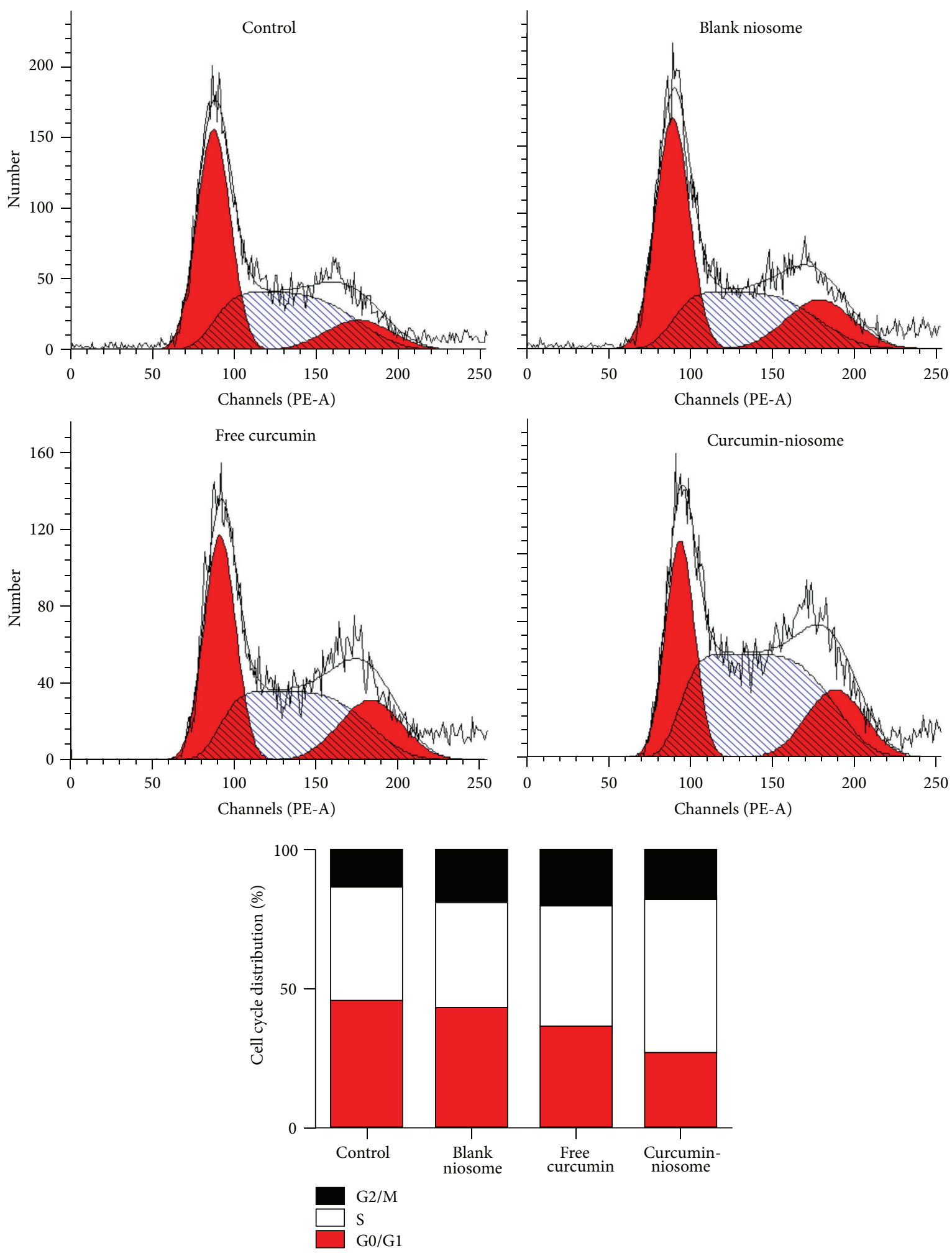

Figure 5: Cell cycle arrest induced by curcumin-niosomes.

used $(25 \mu \mathrm{M})$, while free curcumin killed only $20 \%$ of the cells. Compared to free curcumin, curcumin-niosomes exhibited remarkable cytotoxicity, which is likely due to improved aqueous curcumin solubility and enhanced cellular uptake.
3.5. Cell Cycle Arrest. The growth inhibition mechanisms of curcumin-niosome in ovarian cancer A2780 cells were investigated using cell cycle analyses. Analyses were performed on A2780 cells after $24 \mathrm{~h}$ of drug incubation using flow cytometry (Figure 5). The blank niosomes and control group 
displayed similar cell cycle distribution, while curcuminniosomes increased the fraction of cells in $\mathrm{S}$ phase. The enhanced effect was observed to be $43.74 \%$ in the cells treated with $12.5 \mu \mathrm{M}$ of curcumin-niosomes and was $55.72 \%$ in the cells treated with equivalent concentration of free curcumin (Figure 5). This suggests curcumin-niosomes significantly arrest the cell cycle of ovarian cancer cells at $\mathrm{S}$ phase.

3.6. Proapoptotic Effects. Induction of apoptosis is an effective strategy used in cancer chemotherapy. It was previously reported that curcumin was capable of inducing apoptosis in ovarian cancer cells [2]. In order to investigate the proapoptotic effect of curcumin-niosomes, the curcurmin-niosomes induced apoptosis was characterized by Annexin V/PI dual staining. For early apoptotic cells, phosphatidylserine (PS) turns from the inner side of the cell membrane to the outer leaflet, which is an important indication of early apoptosis [28]. Since Annexin V has a high affinity to PS, early apoptotic cells can therefore be detected by Annexin V staining [29]. As shown in Figure 6, the apoptotic rate of A2780 cells after $24 \mathrm{~h}$ treatment was $2.5 \%$ and $4.65 \%$ in the control and blank niosome group, respectively, while free curcumin $(12.5 \mu \mathrm{M})$ induced A2780 cell apoptosis at a ratio of $12.85 \%$ and the curcumin-niosomes $(12.5 \mu \mathrm{M})$ induced an apoptotic ratio of $42.7 \%$. These results indicate that the curcumin-niosomes induced apoptosis more effectively than the freely dispersed curcumin.

\section{Discussion}

Ovarian cancer is one of the leading causes of death in gynecological malignancies. Yet, the successful treatment of this cancer remains elusive accounting for several impediments including a lack in early diagnosis due to a symptomless early state, a high incidence of recurrence, and chemoresistance $[30,31]$. The development of effective therapeutic agents for ovarian cancer therapy remains a key goal of current cancer therapy research. Curcumin has garnered increased interest due to its multiple bioactive effects including antiapoptosis, anti-inflammation, antiangiogenesis, and chemosensitivity. However, the poor aqueous solubility and rapid metabolism of curcumin have greatly impeded its clinical usage $[1,6]$. Moreover, curcumin suffers from self-degradation even in the dark at physiological $\mathrm{pH}$, while it is also photodegradable due to its light sensitivity in phosphate buffer ( $\mathrm{pH} 7.4)$ [32]. To this end, various particles, such as biocompatible and biodegradable liposomes, have been used to improve the stability of curcumin and ferry curcumin to cancer cells. Karewicz et al. prepared curcumin-loaded liposomes consisting of dihexyl phosphate (DPH), egg yolk phosphatidylcholine (EYPC), and cholesterol by the film evaporation technique, allowing curcumin to be soluble in the lipophilic bilayer owing to its lipophilicity. EYPC/DPH/cholesterol liposomal bilayer enabled improving the stability of curcumin and served as a favorable carrier for curcumin [33].

Niosomes are nonionic surfactant vesicles composed of nonionic surfactants such as Span 80, Span 60, Span 40, and
Span 20, Tween 80, Pluronic 188, cholesterol, and Cremophor RH40. Niosomes have a similar bilayer structure as liposomes but are more cost-effective and have better chemical and storage capabilities owing to the easy derivatization and versatile vesicular structure of surfactants. Taking advantage of niosomes, $\mathrm{pH}$-sensitive niosomal formulations composed of PEG-poly(monomethyl itaconate)-CholC6 and cholesteryl hemisuccinate were reported to be prepared by an ethanol injection method and were evidenced to improve the in vitro effect and to reduce the side effects of the loaded drug [34].

Considering the merits of niosome overwhelming liposome, we constructed a niosome-based system for curcumin delivery to improve its cytotoxic efficacy. In the present study, a novel niosome system composed of the nonionic surfactants, Span 80, Tween 80 , and Poloxamer 188, has been developed. Curcumin was encapsulated in these niosomes with a high entrapment efficiency of $92.3 \pm 0.4 \%$, twice the reported curcumin-loaded Lipo-PEG-PEI complex (45\%) [35]. The DLS analysis revealed that the mean diameter of curcumin-niosomes was $84.15 \pm 4.03 \mathrm{~nm}$ at the homogenization pressure of 800 bar and exhibited superior size compared to other curcumin-loaded liposomes $(157 \mathrm{~nm}$ for curcumin-loaded silica-coated flexible liposomes, $132 \mathrm{~nm}$ for curcumin-loaded liposomes coupled with ApoE peptide, and $207 \mathrm{~nm}$ for curcumin-conjugated nanoliposomes) [3638]. Curcumin-niosomes with considerable size $(84.15 \mathrm{~nm})$ were expected to accumulate in tumor tissues via enhanced permeability and retention. Interestingly, the particle size of curcumin-niosomes was found to be slightly smaller than its blank niosomes counterpart, which was attributed to the excellent binding between curcumin's lipophilic chemical structures to the lipophilic segments of surfactant. Moreover, the accumulated release of curcumin from niosomes reached $80 \%$ after $24 \mathrm{~h}$, suggesting that niosomes provide excellent release from the carriers.

The blank niosomes did not induce cytotoxicity on cells, suggesting the niosomes had high biocompatibility and were suitable for drug delivery system. Curcumin-niosomes demonstrated enhanced cytotoxicity and proapoptotic effects when compared to free curcumin treatment of the A2780 cancer cell line, indicating that niosomes promoted cellular uptake and the protection of the bioactive, but biochemically unstable chemical, curcumin. This was evidenced by increased intracellular levels of curcumin (1.43-fold) through niosomes-mediated delivery compared to that of freely dispersed curcumin after $4 \mathrm{~h}$ incubation. Cell cycle analyses were also performed after $24 \mathrm{~h}$ treatment to investigate the growth inhibition mechanism of curcumin-niosomes. The analyses demonstrated significant cell cycle arrest at $S$ phase of ovarian cancer cells treated with curcumin-niosomes, further confirming the enhanced apoptotic effects of curcuminniosomes. These results demonstrate that niosomes represent a promising drug delivery system for curcumin in cancer therapy. In addition, niosomes can be further functionalized to specifically target tumor sites and prolong circulation in the body. The in vivo antitumor effects of curcumin-niosomes are still under investigation and will be the subject in subsequent studies. 

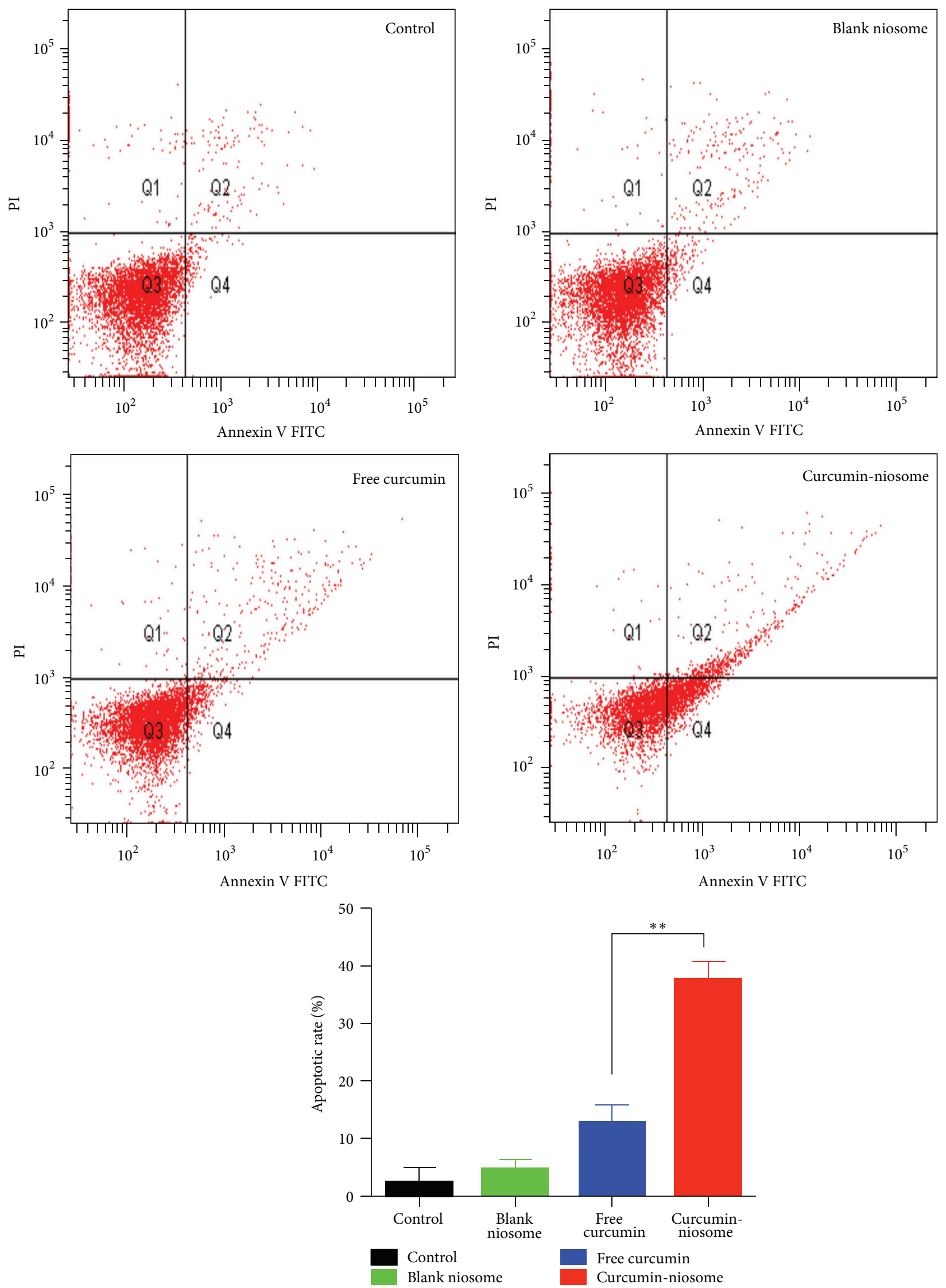

Figure 6: Apoptotic effects induced by curcumin-niosomes $\left({ }^{* *} p<0.01\right)$. 


\section{Conclusions}

In this study, a novel curcumin-loaded niosomes system was developed to effectively deliver curcumin for the treatment of ovarian cancer. It was demonstrated that niosomes provided a biochemically stable protection and high entrapment efficiency of curcumin. Moreover, curcumin-niosomes exhibited enhanced cellular uptake, cytotoxic activity, cell cycle arrest, and apoptotic rate against ovarian cancer A2780 cells. Taken together, curcumin-niosomes are potential delivery formulations for the treatment of ovarian cancer.

\section{Competing Interests}

The authors declare that there are no competing interests regarding the publication of this paper.

\section{Authors' Contributions}

Ying-Qi Xu and Wen-Rong Chen contributed equally to the work.

\section{Acknowledgments}

This study was supported by the Macao Science and Technology Development Fund (077/2011/A3), the Research Fund of the University of Macau (MYRG2014-00033-ICMSQRCM, MYRG2014-00051-ICMS-QRCM, and MYRG201500171-ICMS-QRCM), and the National Natural Science Foundation of China (81403120).

\section{References}

[1] O. Naksuriya, S. Okonogi, R. M. Schiffelers, and W. E. Hennink, "Curcumin nanoformulations: a review of pharmaceutical properties and preclinical studies and clinical data related to cancer treatment," Biomaterials, vol. 35, no. 10, pp. 3365-3383, 2014.

[2] N. G. Vallianou, A. Evangelopoulos, N. Schizas, and C. Kazazis, "Potential anticancer properties and mechanisms of action of curcumin," Anticancer Research, vol. 35, no. 2, pp. 645-651, 2015.

[3] C. N. Sreekanth, S. V. Bava, E. Sreekumar, and R. J. Anto, "Molecular evidences for the chemosensitizing efficacy of liposomal curcumin in paclitaxel chemotherapy in mouse models of cervical cancer," Oncogene, vol. 30, no. 28, pp. 3139-3152, 2011.

[4] M. Heger, R. F. van Golen, M. Broekgaarden, and M. C. Michel, "The molecular basis for the pharmacokinetics and pharmacodynamics of curcumin and its metabolites in relation to cancers," Pharmacological Reviews, vol. 66, no. 1, pp. 222-307, 2014.

[5] A. Shehzad, F. Wahid, and Y. S. Lee, "Curcumin in cancer chemoprevention: molecular targets, pharmacokinetics, bioavailability, and clinical trials," Archiv der Pharmazie, vol. 343, no. 9, pp. 489-499, 2010.

[6] N. M. Khalil, T. C. F. D. Nascimento, D. M. Casa et al., "Pharmacokinetics of curcumin-loaded PLGA and PLGA-PEG blend nanoparticles after oral administration in rats," Colloids and Surfaces B: Biointerfaces, vol. 101, pp. 353-360, 2013.

[7] P. Anand, A. B. Kunnumakkara, R. A. Newman, and B. B. Aggarwal, "Bioavailability of curcumin: problems and promises," Molecular Pharmaceutics, vol. 4, no. 6, pp. 807-818, 2007.
[8] S. Barui, S. Saha, G. Mondal, S. Haseena, and A. Chaudhuri, "Simultaneous delivery of doxorubicin and curcumin encapsulated in liposomes of pegylated RGDK-lipopeptide to tumor vasculature," Biomaterials, vol. 35, no. 5, pp. 1643-1656, 2014.

[9] X. Zhao, Q. Chen, W. Liu et al., "Codelivery of doxorubicin and curcumin with lipid nanoparticles results in improved efficacy of chemotherapy in liver cancer," International Journal of Nanomedicine, vol. 10, pp. 257-270, 2015.

[10] K. Nagahama, Y. Sano, and T. Kumano, "Anticancer drug-based multifunctional nanogels through self-assembly of dextrancurcumin conjugates toward cancer theranostics," Bioorganic \& Medicinal Chemistry Letters, vol. 25, no. 12, pp. 2519-2522, 2015.

[11] S. P. Singh, M. Sharma, and P. K. Gupta, "Cytotoxicity of curcumin silica nanoparticle complexes conjugated with hyaluronic acid on colon cancer cells," International Journal of Biological Macromolecules, vol. 74, pp. 162-170, 2015.

[12] A. Kumar, A. Ahuja, J. Ali, and S. Baboota, "Curcumin loaded nano globules for solubility enhancement: preparation, characterization and ex vivo release study," Journal of Nanoscience and Nanotechnology, vol. 12, no. 11, pp. 8293-8302, 2012.

[13] X. Xie, A. M. Xu, S. Leal-Ortiz, Y. Cao, C. C. Garner, and N. A. Melosh, "Nanostraw-electroporation system for highly efficient intracellular delivery and transfection," ACS Nano, vol. 7, no. 5, pp. 4351-4358, 2013.

[14] X. Xie, A. M. Xu, M. R. Angle, N. Tayebi, P. Verma, and N. A. Melosh, "Mechanical model of vertical nanowire cell penetration," Nano Letters, vol. 13, no. 12, pp. 6002-6008, 2013.

[15] M. Chen, Z. Zhong, W. Tan, S. Wang, and Y. Wang, "Recent advances in nanoparticle formulation of oleanolic acid," Chinese Medicine, vol. 6, article 20, 2011.

[16] G. Puras, M. Mashal, J. Zárate et al., "A novel cationic niosome formulation for gene delivery to the retina," Journal of Controlled Release, vol. 174, no. 1, pp. 27-36, 2014.

[17] R. Rajera, K. Nagpal, S. K. Singh, and D. N. Mishra, "Niosomes: a controlled and novel drug delivery system," Biological and Pharmaceutical Bulletin, vol. 34, no. 7, pp. 945-953, 2011.

[18] A. Azeem, M. K. Anwer, and S. Talegaonkar, "Niosomes in sustained and targeted drug delivery: some recent advances," Journal of Drug Targeting, vol. 17, no. 9, pp. 671-689, 2009.

[19] D. Cosco, D. Paolino, R. Muzzalupo et al., "Novel PEG-coated niosomes based on bola-surfactant as drug carriers for 5fluorouracil," Biomedical Microdevices, vol. 11, no. 5, pp. 1115$1125,2009$.

[20] G. Abdelbary and N. El-Gendy, "Niosome-encapsulated gentamicin for ophthalmic controlled delivery," AAPS PharmSciTech, vol. 9, no. 3, pp. 740-747, 2008.

[21] N. Yuksel, Z. S. Bayindir, E. Aksakal, and A. T. Ozcelikay, "In situ niosome forming maltodextrin proniosomes of candesartan cilexetil: in vitro and in vivo evaluations," International Journal of Biological Macromolecules, vol. 82, pp. 453-463, 2016.

[22] M. Najlah, K. Hidayat, H. K. Omer et al., "A facile approach to manufacturing non-ionic surfactant nanodipsersions using proniosome technology and high-pressure homogenization," Journal of Liposome Research, vol. 25, no. 1, pp. 32-37, 2015.

[23] Y.-Z. Zhao, C.-T. Lu, Y. Zhang et al., "Selection of high efficient transdermal lipid vesicle for curcumin skin delivery," International Journal of Pharmaceutics, vol. 454, no. 1, pp. 302309, 2013.

[24] C. F. Chignell, P. Bilski, K. J. Reszka, A. G. Motten, R. H. Sik, and T. A. Dahl, "Spectral and photochemical properties of curcumin," Photochemistry and Photobiology, vol. 59, no. 3, pp. 295-302, 1994. 
[25] S. Wang, R. Chen, Z. Zhong, Z. Shi, M. Chen, and Y. Wang, "Epigallocatechin-3-gallate potentiates the effect of curcumin in inducing growth inhibition and apoptosis of resistant breast cancer cells," American Journal of Chinese Medicine, vol. 42, no. 5, pp. 1279-1300, 2014.

[26] J.-L. Gao, T.-C. He, Y.-B. Li, and Y.-T. Wang, "A traditional Chinese medicine formulation consisting of Rhizoma Corydalis and Rhizoma Curcumae exerts synergistic anti-tumor activity," Oncology Reports, vol. 22, no. 5, pp. 1077-1083, 2009.

[27] X.-Y. Ke, V. W. Lin Ng, S.-J. Gao, Y. W. Tong, J. L. Hedrick, and Y. Y. Yang, "Co-delivery of thioridazine and doxorubicin using polymeric micelles for targeting both cancer cells and cancer stem cells," Biomaterials, vol. 35, no. 3, pp. 1096-1108, 2014.

[28] L. Ouyang, Z. Shi, S. Zhao et al., "Programmed cell death pathways in cancer: a review of apoptosis, autophagy and programmed necrosis," Cell Proliferation, vol. 45, no. 6, pp. 487498, 2012.

[29] S. Wang, R. Chen, J. Morott, M. A. Repka, Y. Wang, and M. Chen, "MPEG-b-PCL/TPGS mixed micelles for delivery of resveratrol in overcoming resistant breast cancer," Expert Opinion on Drug Delivery, vol. 12, no. 3, pp. 361-373, 2015.

[30] Á. H. I. Garces, M. S. F. Dias, E. Paulino, C. G. M. Ferreira, and A. C. De Melo, "Treatment of ovarian cancer beyond chemotherapy: are we hitting the target?" Cancer Chemotherapy and Pharmacology, vol. 75, no. 2, pp. 221-234, 2015.

[31] S. Carpi, S. Fogli, A. Giannetti et al., "Theranostic properties of a survivin-directed molecular beacon in human melanoma cells," PLoS ONE, vol. 9, no. 12, article el14588, 2014.

[32] S. Mondal, S. Ghosh, and S. P. Moulik, "Stability of curcumin in different solvent and solution media: UV-visible and steadystate fluorescence spectral study," Journal of Photochemistry and Photobiology B: Biology, vol. 158, pp. 212-218, 2016.

[33] A. Karewicz, D. Bielska, B. Gzyl-Malcher, M. Kepczynski, R. Lach, and M. Nowakowska, "Interaction of curcumin with lipid monolayers and liposomal bilayers," Colloids and Surfaces B: Biointerfaces, vol. 88, no. 1, pp. 231-239, 2011.

[34] S. Ghanbarzadeh, A. Khorrami, Z. Pourmoazzen, and S. Arami, "Plasma stable, pH-sensitive non-ionic surfactant vesicles simultaneously enhance antiproliferative effect and selectivity of Sirolimus," Pharmaceutical Development and Technology, vol. 20, no. 3, pp. 279-287, 2015.

[35] Y.-L. Lin, Y.-K. Liu, N.-M. Tsai et al., "A Lipo-PEG-PEI complex for encapsulating curcumin that enhances its antitumor effects on curcumin-sensitive and curcumin-resistance cells," Nanomedicine: Nanotechnology, Biology, and Medicine, vol. 8, no. 3, pp. 318-327, 2012.

[36] C. Li, Y. Zhang, T. T. Su, L. Feng, Y. Long, and Z. Chen, "Silicacoated flexible liposomes as a nanohybrid delivery system for enhanced oral bioavailability of curcumin," International Journal of Nanomedicine, vol. 7, pp. 5995-6002, 2012.

[37] F. Re, I. Cambianica, C. Zona et al., "Functionalization of liposomes with ApoE-derived peptides at different density affects cellular uptake and drug transport across a blood-brain barrier model," Nanomedicine: Nanotechnology, Biology, and Medicine, vol. 7, no. 5, pp. 551-559, 2011.

[38] A. N. Lazar, S. Mourtas, I. Youssef et al., "Curcumin-conjugated nanoliposomes with high affinity for $\mathrm{A} \beta$ deposits: possible applications to Alzheimer disease," Nanomedicine: Nanotechnology, Biology, and Medicine, vol. 9, no. 5, pp. 712-721, 2013. 

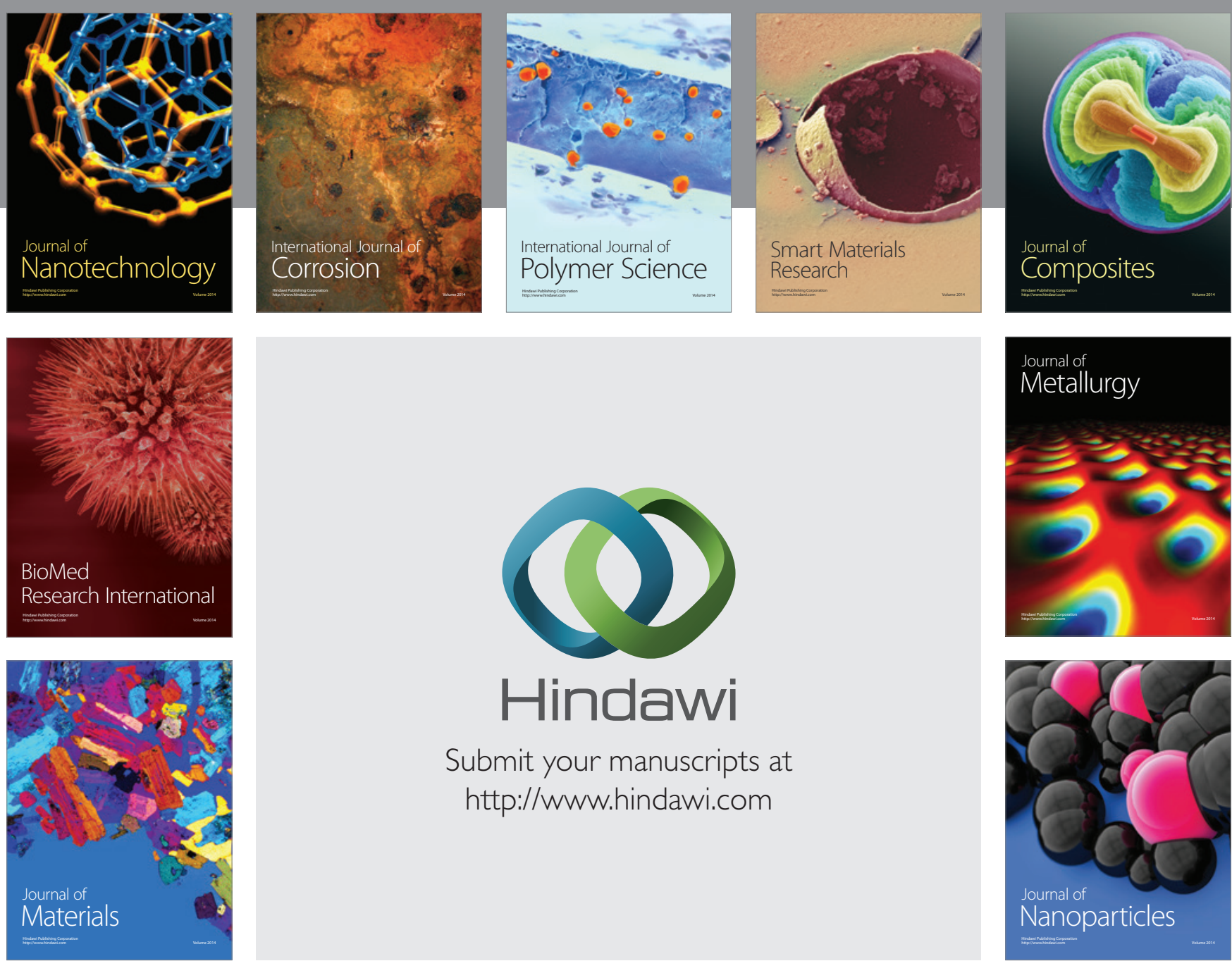

\section{Hindawi}

Submit your manuscripts at

http://www.hindawi.com

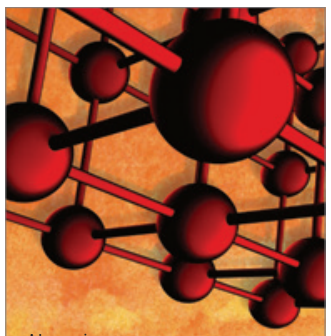

Materials Science and Engineering
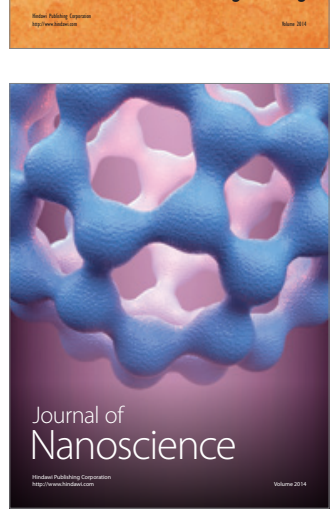
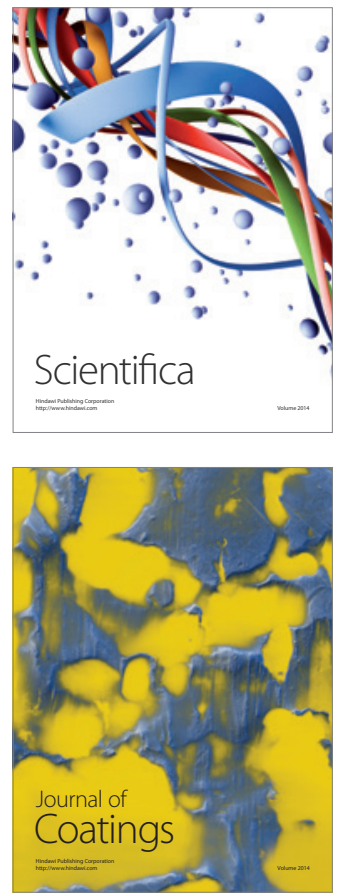
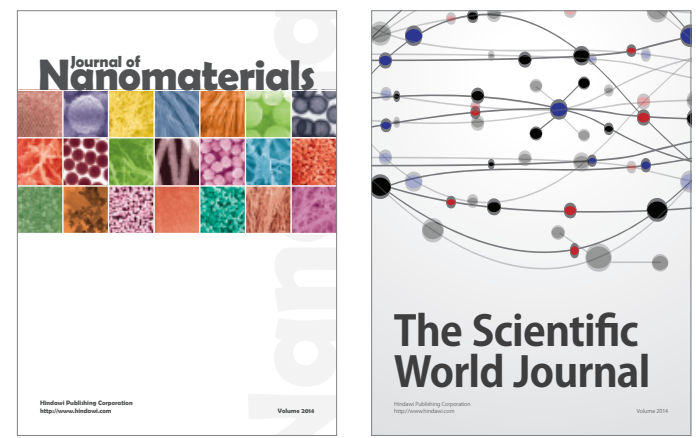

The Scientific World Journal
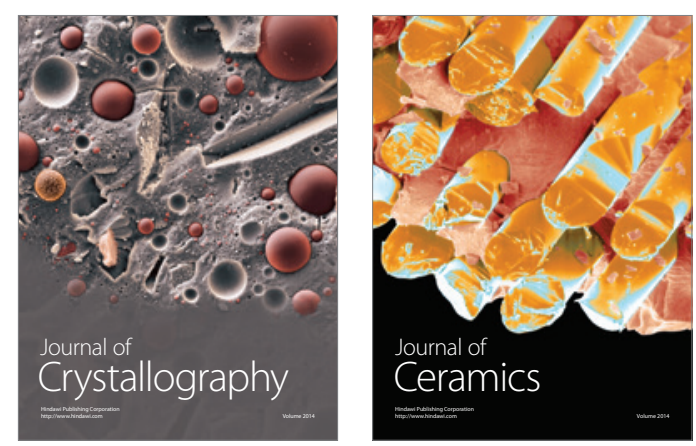
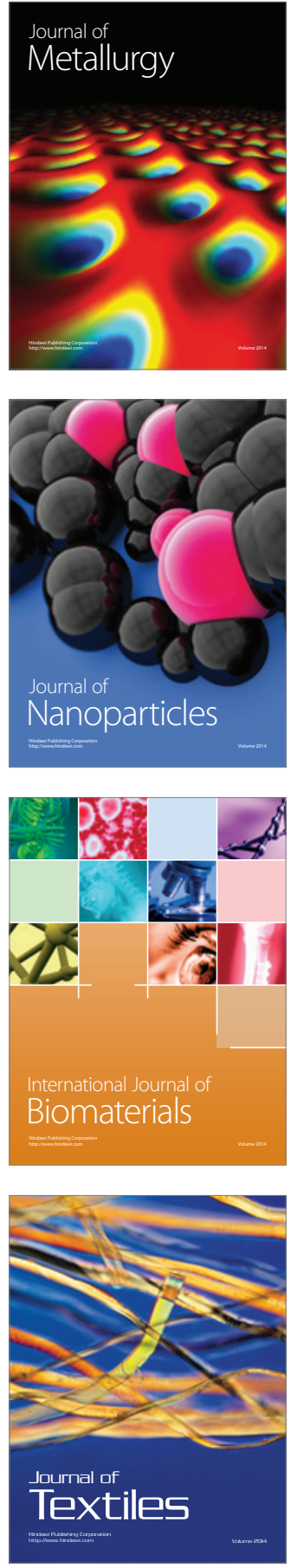